Article

\title{
Attenuation Correction for Ka-band Cloud Radar Using X-band Weather Radar Data
}

Peng Zhang ${ }^{1, *}$ and Yunjie Chen ${ }^{2}$

1 Institute of Meteorology and Ocean, PLA University of Science and Technology, Nanjing 211101, China

2 Jiangsu Key Laboratory of Meteorological Observation and Information Processing, Nanjing University of Information Science and Technology, Nanjing 210044, China

* Correspondence: zhangpeng_meteo@yahoo.com; Tel.: (+86)02580820542, Fax: (+86)02580820542

\begin{abstract}
In order to correct attenuated millimeter-wavelength (Ka-band) radar data and address the problem of instability, an attenuation correction methodology (attenuation correction with variation trend constraint; VTC) was developed. Using synchronous observation conditions and multi-band radars, the VTC method adopts the variation trends of reflectivity in X-band radar data captured with wavelet transform as a constraint to adjust reflectivity factors of millimeter-wavelength radar. The correction was evaluated by comparing reflectivities obtained by millimeter-wavelength cloud radar and X-band weather radar. Experiments showed that attenuation was a major contributory factor in the different reflectivities of the two radars when relatively intense echoes exist, and the attenuation correction developed in this study significantly improved data quality for millimeter-wavelength radar. Reflectivity differences between the two radars were reduced and reflectivity correlations were enhanced. Errors caused by attenuation were eliminated, while variation details in the reflectivity factors were retained. The VTC method is superior to the bin-by-bin method in terms of correction amplitude and can be used for attenuation correction of shorter wavelength radar assisted by longer wavelength radar data.
\end{abstract}

Keywords: millimeter-wavelength cloud radar; attenuation correction; dual-radar; data fusion

\section{Introduction}

The role of clouds in Earth-atmosphere radiation transmission and balance is an important factor that affects climate change [1]. Furthermore, it is also a key factor for weather forecasting and for performing artificial weather modification. Therefore, the observation and analysis of macro and micro cloud parameters is important. Compared with centimeter-wavelength radar, millimeter-wavelength cloud radar (MMCR) has higher sensitivity, a larger dynamic range, smaller antenna size, lower transmitted peak power, and finer temporal-spatial resolution; thus, MMCR represents an effective means for cloud observation [e.g., 2, 3]. MMCR bridges an observational gap in Earth's hydrological cycle by adequately detecting clouds and precipitation [4], and has been employed by a number of studies [e.g., 
5-11]. However, the data from MMCR working at $35 \mathrm{GHz}$ (Ka-band) and $94 \mathrm{GHz}$ (W-band) are usually significantly more attenuated than centimeter-wavelength radar data [3]. The intensities and areas of echoes are degraded and clouds or precipitation behind intense echoes cannot be detected. Attenuation errors in raw data are transferred into sequential quantitative productions; therefore, it is vital to analyze and correct attenuation errors in order to allow for further quantitative applications.

Lhermitte [3] studied the attenuation and scattering of millimeter-wavelength radiation by clouds and precipitation. So far, most of the available attenuation correction methods are modified from those used with centimeter-wavelength radar. For conventional radar, empirical relationships between specific attenuation $\left(A_{H}\right)$ and the radar reflectivity factor $\left(Z_{H}\right)$ have been adopted for the correction of reflectivity [12, 13]; however, because $A_{H}$ is calculated according to measured (or attenuated) rather than intrinsic (or unattenuated) $Z_{H}$, methods of this kind are inherently unstable [13] and can lead to over-correction or insufficient correction. In order to address this problem, numerous constraints on the attenuation correction procedure have been considered. Surface reference technology (SRT) [e.g., 14, 15] obtains path-integrated attenuation (PIA) by calculating the differences between the reflectivities of precipitation and reference echoes (e.g., mountain echo under clear sky conditions), while dual-wavelength methods obtain PIA by comparing the reflectivities of radars at different wavelengths $[15,16]$. Seto [17] modified the $A_{H}-Z_{H}$ relationship by using the dual-frequency ratio (DFR) of $Z_{H}$ and SRT for dual-frequency precipitation radar (DPR) on the core satellite of the Global Precipitation Measurement (GPM) mission. However, the accuracy of these methods is significantly influenced by the reflectivity accuracy of the reference echo.

For dual-polarized radar, PIA can be estimated using the total differential propagation phase $\left(\phi_{D P}\right)$ based on the approximate linear relationship between $A_{H}$ and the specific differential phase $\left(K_{D P}\right)$ [e.g., 18-20]; the proportionality factor between $A_{H}$ and $K_{D P}$ is very sensitive to temperature, drop size distribution (DSD), and drop shape variations [18, 21]. Finally, the self-consistent method with constraints was devised to estimate the optimal $A_{H}-K_{D P}$ relationship $[22,23]$. However, $K_{D P}$ can be heavily polluted by noise, in particular light rainfall [e.g., 23-25], and so for MMCR whose main targets are clouds and light rainfall, correction methods based on $K_{D P}$ may introduce significant additional error.

The existing correction methods described above rely on the radar itself and have numerous defects. As an alternative, attenuation correction methods assisted by other radars or instruments have been developed. Stereoradar methods were developed to retrieve reflectivity by using the data from two radars [26]. Radiometer measurements have been introduced into the correction of radar reflectivity [27]. PIA obtained by microwave link has also been used [e.g., 28-31]. In networked radar environments, reflectivity and specific attenuation along each 
beam have been obtained by combined backscatter and forward scatter equations of the precipitation medium with constraints on PIA [32].

With the current level of development for meteorological radar, it is becoming more common for multiple cloud and precipitation observation radars to be working concurrently, using the same or different wavelengths and covering overlapping observation areas. This situation is especially common in countries and regions where centimeter-wavelength weather radar networks have been established and mosaic productions of network-wide radars are available (e.g., China, Europe, the United States).

In this study, a new method for reducing the error introduced by attenuation of MMCR data was developed. The attenuation correction with variation trend constraint (VTC) method compensates for the reflectivities of MMCR by using the variation trend of reflectivities in X-band weather radar (XR) as a constraint. Analyzed radar data showed that the VTC method can effectively reduce attenuation errors in the reflectivities of MMCR, while also addressing instability problems and maintaining variation details.

\section{Materials and Methods}

2.1 Evaluating attenuation impact on MMCR reflectivity

Attenuation originates from absorption and scattering. For MMCR, attenuation mainly includes two parts: atmospheric absorption due to oxygen and water vapor, and attenuation due to liquid water [3]. Atmospheric absorption can be calculated according to the Stepanenko absorption model [33]. Since MMCR usually works at the Ka-band (35 GHz) and W-band (95 GHz), both of which are atmospheric windows, attenuation due to atmospheric absorption is negligible and can be ignored; therefore, only attenuation caused by water vapor in clouds needs to be considered.

To analyze the impacts of attenuation on the reflectivity of MMCR, with an aim to optimize and evaluate an attenuation correction methodology, a comparative analysis of reflectivities was conducted. Radar data were collected from two radars (Table 1): a Ka-band ground-based MMCR and an XR used for weather surveillance. These radars were working synchronously in the World Meteorological Organization $(\mathrm{WMO}) 8^{\text {th }}$ radiosondes inter-comparison and integrated remote instruments experiment, conducted in July 2010 in Guangzhou Province, China. 
Table1. Main characteristics of the MMCR and XR ${ }^{a}$

\begin{tabular}{|c|c|c|c|c|c|}
\hline Radar & $\begin{array}{l}\text { Wavelength } \\
/ \mathrm{cm}\end{array}$ & $\begin{array}{c}\text { Gate } \\
\text { width } \\
/ \mathrm{m} \\
\end{array}$ & $\begin{array}{c}\text { Antenna } \\
\text { beam } \\
\text { width / }\end{array}$ & $\begin{array}{l}\text { Range } \\
/ \mathrm{km}\end{array}$ & $\begin{array}{c}\text { Peak } \\
\text { power } \\
\text { /w }\end{array}$ \\
\hline MMCR & 0.86 & 50 & 0.4 & 30 & 699 \\
\hline XR & 3.2 & 62.5 & 0.99 & 62.5 & 56975 \\
\hline
\end{tabular}

a. Millimeter-wavelength cloud radar (MMCR) and X-band weather radar of the World Meteorological Organization (WMO) $8^{\text {th }}$ radiosondes inter-comparison and integrated remote instruments experiment (July 2010, Guangzhou Province, China)

The MMCR and the XR were situated less than $20 \mathrm{~m}$ apart, and the post-calibration system bias of both was less than $1 \mathrm{~dB}$. In order to eliminate random noise error and the impact of clutter, quality control was performed on the reflectivity data before analysis and correction.

When the sizes of the particles are significantly smaller than wavelength, the Rayleigh scattering approximation is valid and the reflectivity factor can be expressed as:

$$
Z_{H}\left(m m^{6} m^{-3}\right)=\frac{\lambda^{4}}{\pi^{5}\left|k_{w}\right|^{2}} \int \sigma_{H}(D) N(D) d D
$$

where $\lambda$ is the wavelength, $D$ is the volume equivalent spherical diameter, $\sigma_{H}(D)$ represents the radar cross section at horizontal polarization, $N(D)$ is the number of water drops per unit volume per unit size interval, and $k_{w}$ is the dielectric factor of water. The reflectivity factor in $d B Z$ can be defined as:

$$
Z_{H}(d B Z)=10 \log _{10} Z_{H}\left(m m^{6} m^{-3}\right)
$$

In these expressions, $Z_{H}$ is independent of radar wavelength and is controlled by the DSD in the resolution volume. However, in reality the reflectivity factors of the same target observed by two radars at different wavelengths may be different because of factors including attenuation, non-Rayleigh scattering, differences in resolution volumes, system errors, and random error. Among these, system and random errors can be removed by calibration and quality control. For the non-Rayleigh scattering effect, Nakamura [34] and Chandrasekar [35] analyzed the difference between the equivalent reflectivity factor and the reflectivity factor, while Lhermitte [3] showed that for precipitation with a rainrate of $10 \mathrm{~mm} / \mathrm{h}$, reflectivity factors measured by centimeter-wavelength radar and Ka-band radar are almost the same. For precipitation with a rainrate of $100 \mathrm{~mm} / \mathrm{h}$, the reflectivity factor measured by Ka-band radar is $\sim 40 d B$, while for centimeter-wavelength radar it is $\sim 50 d B$; therefore, the differences in reflectivity factors due to non-Rayleigh scattering are usually less than $10 d B$. Differences in the resolution volumes of the two radars, which result from different beam widths and range 
resolution widths, also result in different reflectivities.

\subsection{VTC development}

Without the impact of attenuation, variation trends in the reflectivity factors of MMCR and XR should generally be identical. This has been indirectly confirmed by numerous studies that took the reflectivities of longer wavelength radar as 'true' values to evaluate attenuation retrieval methods for shorter wavelength radar [eg.36-38]. On this basis, it is reasonable to correct the reflectivity of MMCR for attenuation by using the variation trends in XR reflectivity as constraint. The VTC method was developed in four steps:

Step 1: to enable attenuation correction using data collected by radar at another site, MMCR and XR radar data in constant altitude plan position indicator (CAPPI) were converted from the polar coordinate system into the absolute geographic coordinate system, and from a conical scanning surface to a constant altitude plane.

Step 2: along each constant line of longitude or latitude in the overlap region, the reflectivity profiles of both radars were decomposed at different scales using wavelet analysis in order to produce the low frequency component $\left(C A_{m m}\right)$ and high frequency component $\left(C D_{m m}\right)$ of the MMCR reflectivities, and the low frequency component of the $\mathrm{XR}$ reflectivities $\left(C A_{X}\right)$.

Step 3:a weighted summation method was applied to adjust the $C A_{m m}$ with respect to $C A_{X}$ using the expression:

$$
C A_{m m}^{\prime}=W_{m m} \times C A_{m m}+W_{X} \times C A_{X}
$$

where $C A_{m m}^{\prime}$ is the adjusted low frequency component of the MMCR reflectivities, and $W_{m m}$ and $W_{X}$ are the weight coefficients of $C A_{m m}$ and $C A_{X}$, respectively. In order to preserve variation details in the uncorrected MMCR reflectivities to the greatest extent possible, a weight coefficient selection methodology based on the principal of maximum energy was utilized to determine $W_{m m}$ and $W_{X}$ adaptively. The square sum of wavelet coefficients was calculated using a 5-point sliding window of $C A_{m m}$ and $C A_{X}$ using the expression:

$$
\begin{aligned}
C_{m m i} & =\sum_{j=i-2}^{i+2} C A_{m m j}^{2}, \text { and } \\
C_{X i} & =\sum_{j=i-2}^{i+2} C A_{X j}^{2}
\end{aligned}
$$

where $C A_{m m j}$ and $C A_{X j}$ are the wavelet coefficients of point $j$ in $C A_{m m}$ and $C A_{X}$, respectively. The greater the energy of the wavelet coefficients, the greater the level of detail that will be retained. The weights of point $i$ are calculated by: 


$$
\begin{aligned}
& W_{m m i}=\frac{C_{m m i}}{C_{m m i}+C_{X i}} \text {, and } \\
& W_{X i}=\frac{C_{X i}}{C_{m m i}+C_{X i}}
\end{aligned}
$$

Therefore, at point $i$, equation (3) can be transformed into:

$$
C A_{m m i}^{\prime}=W_{m m i} \times C A_{m m i}+W_{X i} \times C A_{X i}
$$

Step 4:the corrected reflectivity profiles of the MMCR $P_{\left(Z_{m m}\right)}$ were reconstructed with the adjusted low frequency component $\left(C A_{m m}^{\prime}\right)$ and the original high frequency component $\left(C D_{m m}\right)$ using the expression:

$$
P_{\left(Z_{m m}\right)}=\operatorname{Re} \text { construction }\left(C A_{m m}^{\prime}, C D_{m m}\right)
$$

\section{Results and Discussion}

\subsection{Attenuation and differences in reflectivity}

Data were selected when the difference between the observation times of the two radars was less than $3 \mathrm{~min}$, and when the difference between observation elevations was less than 0.1ㅇ. From 25 July 2010 to 30 July 2010, there were 116 matching pairs of plan position indicator (PPI) data. Statistical analysis showed that the mean reflectivity factors of the MMCR were significantly lower than that of the XR (by $13.58 d B$ ), with a correlation coefficient of 0.46 (Table 2).

Table2. Differences between reflectivity factors of MMCR and $\mathrm{XR}^{\mathrm{a}}$

\begin{tabular}{cccc}
\hline Radar & \multicolumn{3}{c}{ Reflectivity factors of echoes detected by both radars } \\
\cline { 2 - 4 } MMCR & Mean/dBZ & Std dev/dBZ & Correlation \\
XR & -13.44 & 15.60 & 0.46 \\
& 0.14 & 13.29 &
\end{tabular}

a.Millimeter-wavelength cloud radar (MMCR) and X-band weather radar of the World Meteorological Organization (WMO) $8^{\text {th }}$ radiosondes inter-comparison and integrated remote instruments experiment (July 2010, Guangzhou Province, China)

The locations of the intense echoes detected by the two radars showed good agreement (Figure 1). Compared with the XR, the MMCR detected weaker echoes, and the areas of weak echoes detected near the MMCR were larger. This higher sensitivity enabled the MMCR to observe clouds and weak precipitation (e.g.,

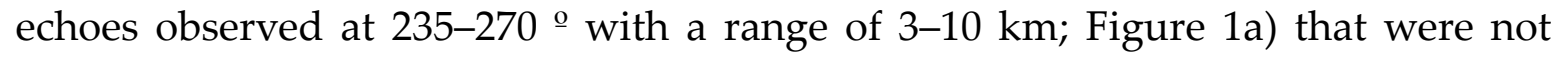
detected by XR. However, reflectivities and areas of intense echoes were smaller than those detected by the $\mathrm{XR}$, in particular on the far side of the radar. Strong attenuation was produced by heavy precipitation; therefore, clouds and/or 
precipitation behind intense echoes were not always detected (e.g., echoes at 20-30 o with a range of 24-30 km; Figure $1 \mathrm{c}$ and d).

(a)

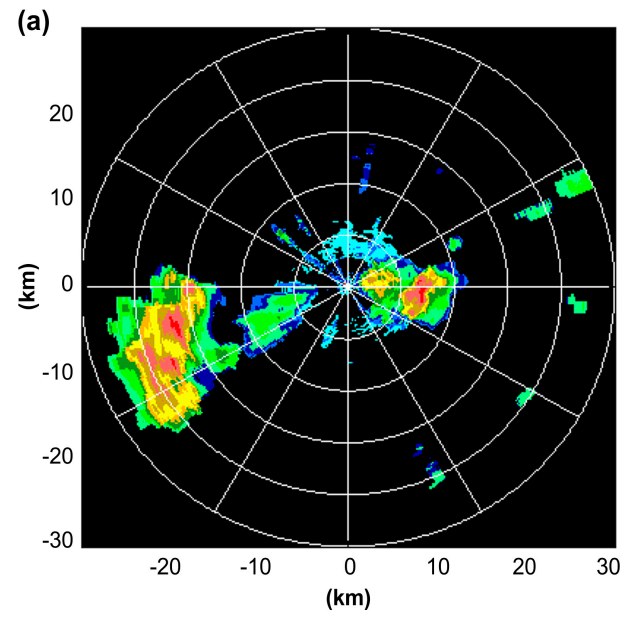

(c)

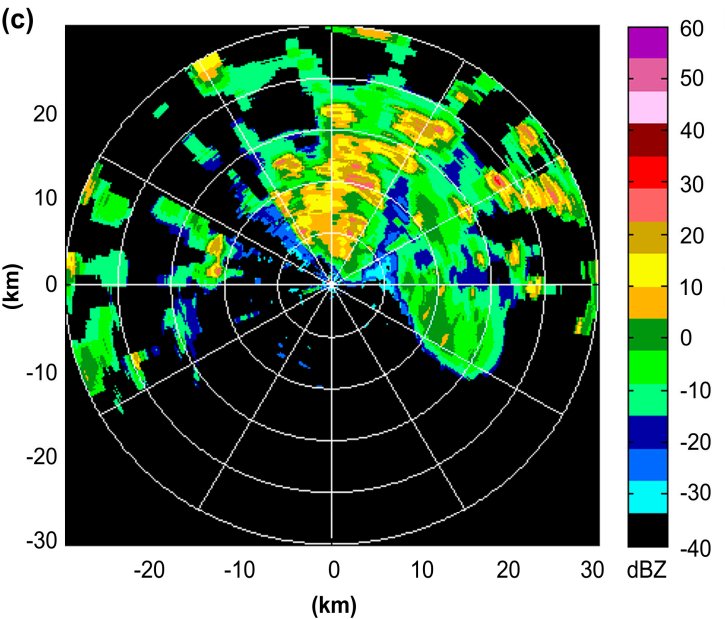

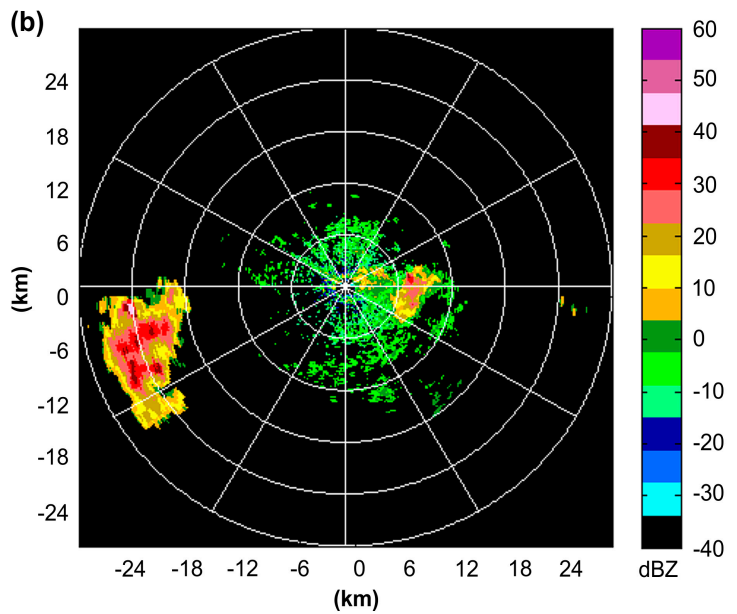

(d)

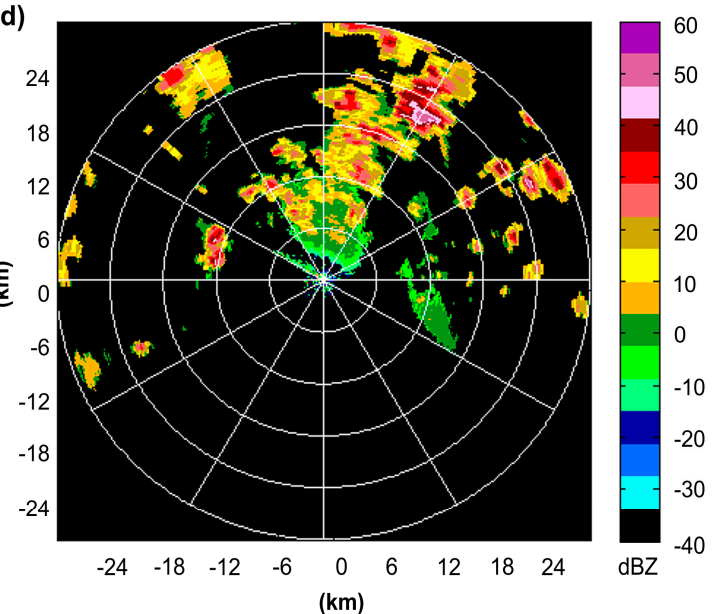

Figure 1. Plan position indicator (PPI) at $6^{\circ}$ elevation in the (a) millimeter-wavelength cloud radar (MMCR) and (b) X-band weather radar (XR) at 13:22 on 26 July 2010, and in the (c) MMCR and (d) XR at 9:18 on 28 July 2010. 

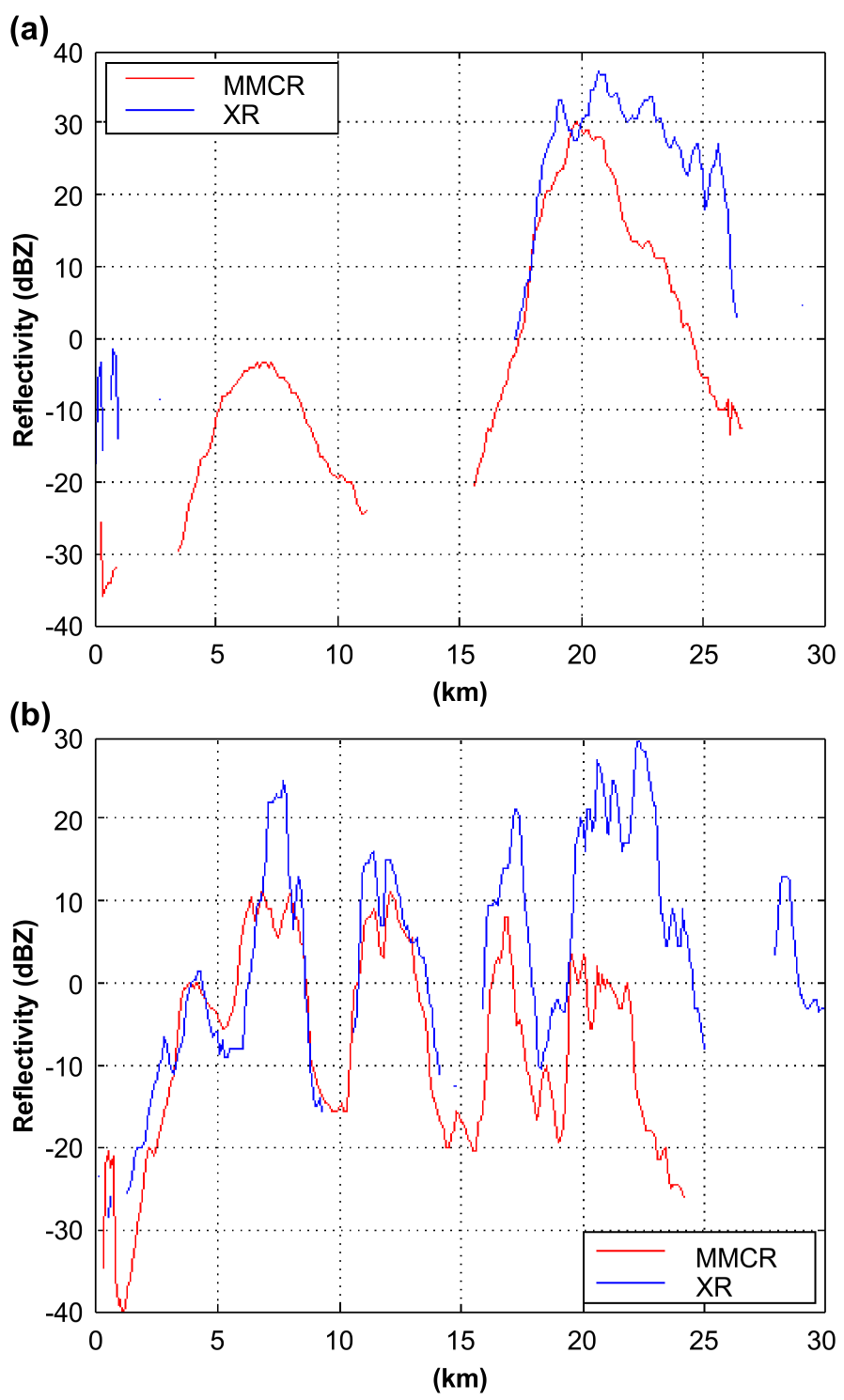

Figure 2. Comparison of radial reflectivities of the millimeter-wavelength cloud radar (MMCR) and X-band weather radar (XR) along the (a) 257 ${ }^{\circ}$ azimuth in Fig. 1a and Fig. 1b, and along the (b) $23^{\circ}$ azimuth in Fig. 1c and Fig. 1d. Breaks in the data represent points where no echo was detected.

The radial reflectivities near the two radars showed good agreement, except for the echoes that only the MMCR detected (Figure 2). As both attenuation and the range increased, the reflectivity differences between the two radars also increased. The largest difference in reflectivity factors was observed at $39 d B$ (Figure 2b).

In contrast with the $\mathrm{XR}$, the reflectivities of the MMCR fell significantly as distance increased, indicating that attenuation had greater impact on reflectivity than resolution volume. More specifically, attenuation always caused the reflectivity to degrade, while differences in the resolution volumes caused both increases and decreases in reflectivity.

In summary, the reflectivities of the MMCR and the XR agreed well when echoes were close, or when there were no intense echoes in the path of electromagnetic wave propagation. In contrast, when echoes were distant from the radars, or when there were intense echoes in the wave propagation path, the 
reflectivities of the MMCR were lower than those of the $\mathrm{XR}$, primarily as a result of attenuation.

\subsection{Experimental outputs}

In order to assess the VTC method, MMCR CAPPI data from 3- $\mathrm{km}$ altitude between 25 and 30 July 2010 were corrected for attenuation (Figure 3). During this time interval, 56 pairs of volume scan data from the two radars had observation times that were less than 3 min apart, and these were used to test the VTC method. The results were compared with those of the bin-by-bin attenuation correction method, in which different $Z_{H}-A_{H}$ relationships were used according to $Z_{H}$ [39].
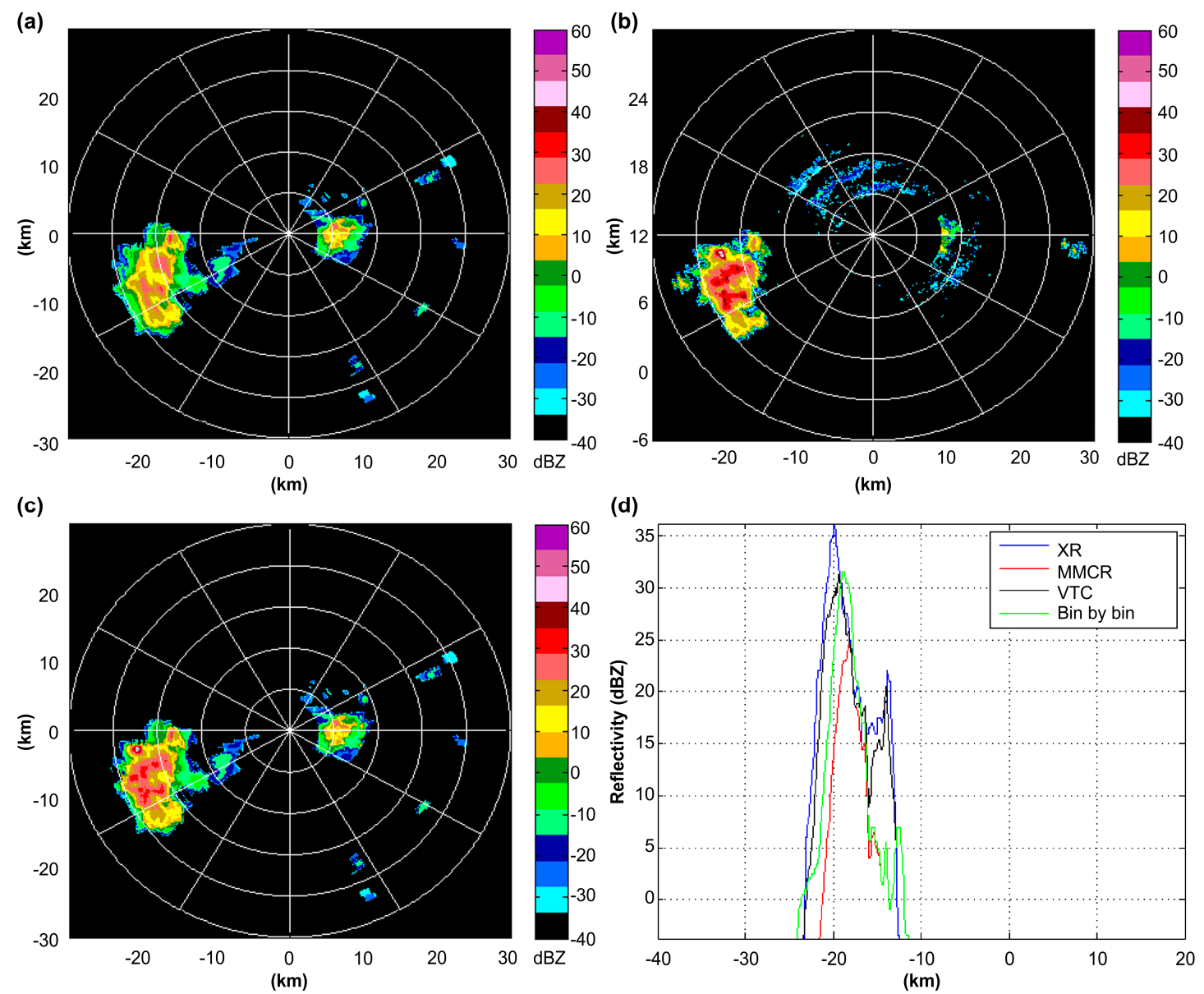

Figure 3. Constant altitude plan position indicator (CAPPI) at 3-km altitude in the 13:22 26 July 2010 reflectivity data of the (a) millimeter-wavelength cloud radar (MMCR), (b) X-band weather radar $(\mathrm{XR})$, and (c)MMCR after the attenuation correction with variation trend constraint (VTC).(d) shows the comparison of MMCR reflectivities before and after VTC correction, along with data from $\mathrm{XR}$ and the MMCR after bin-by-bin correction along a constant line of latitude line $10 \mathrm{~km}$ south of the MMCR. 

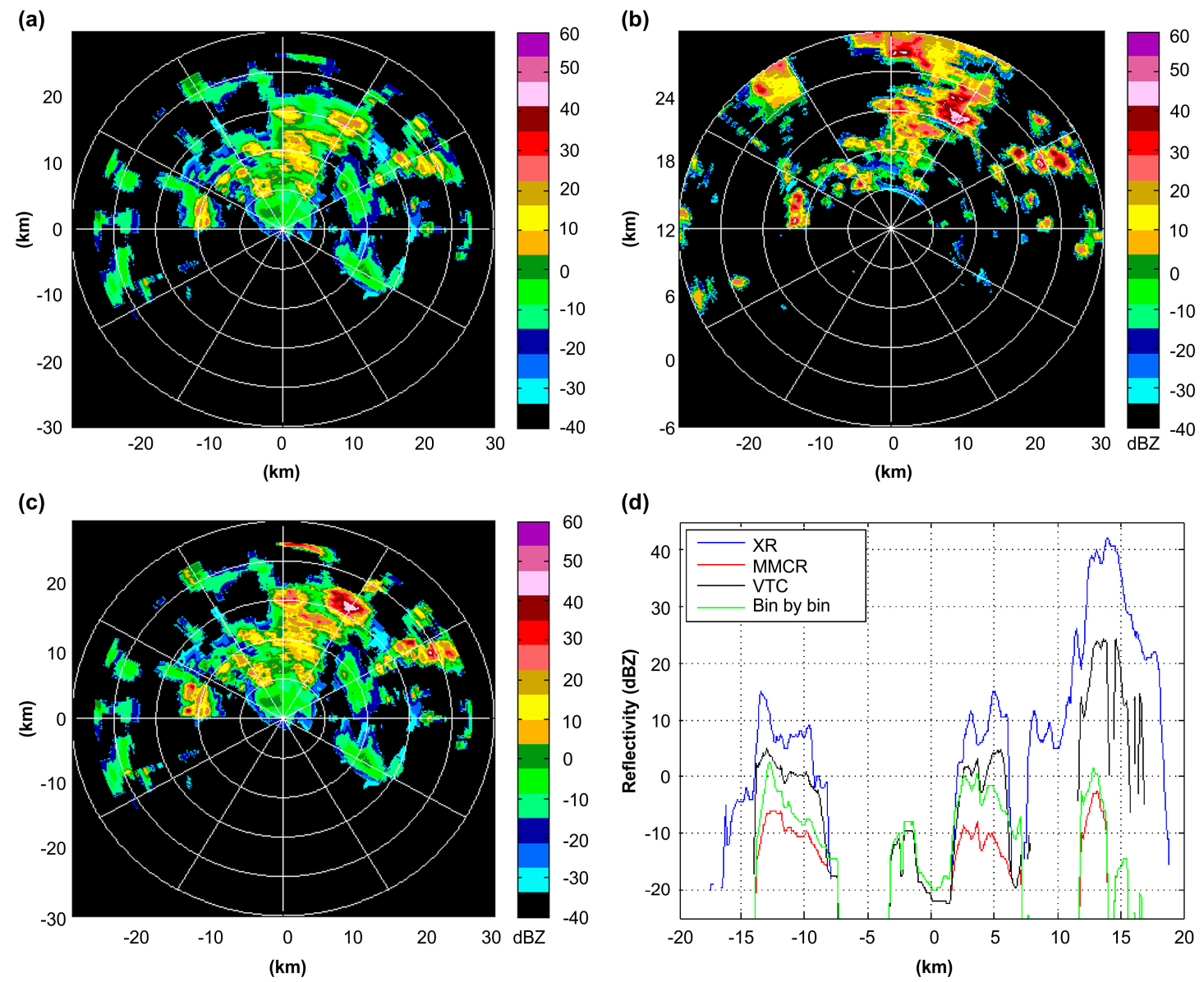

Figure 4.Constant altitude plan position indicator (CAPPI) at $3 \mathrm{~km}$ altitude in the 9:18 28 July 2010 reflectivity data of the (a) millimeter-wavelength cloud radar (MMCR), (b) X-band weather radar (XR), and (c) MMCR after the attenuation correction with variation trend constraint (VTC). (d) shows the comparison of MMCR reflectivities before and after VTC correction, along with data from $\mathrm{XR}$ and the MMCR after bin-by-bin correction along a constant line of latitude line $20 \mathrm{~km}$ north of the MMCR.

The reflectivities and echo areas in the MMCR data became enlarged after correction, such that the echo distribution was closer to that of the XR (Figure 2; Figure 4). Echo degradation was reduced (e.g., the echoes at $20 \mathrm{~km}$ range and

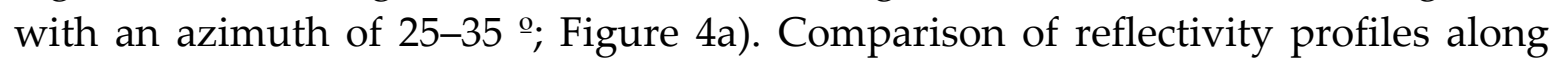
constant lines of latitude (Figure 3d; Figure 4d) showed that the VTC method made the overall variation trends in the MMCR reflectivities closer to those of the XR reflectivities, while still retaining the variation details in the MMCR reflectivities. Furthermore, the correction amplitudes of the VTC method were greater than those of the bin-by-bin method. The maximum correction value reached $27 d B$ (Figure $4 \mathrm{~d}$ ), which is consistent with the findings of our previous analysis. 

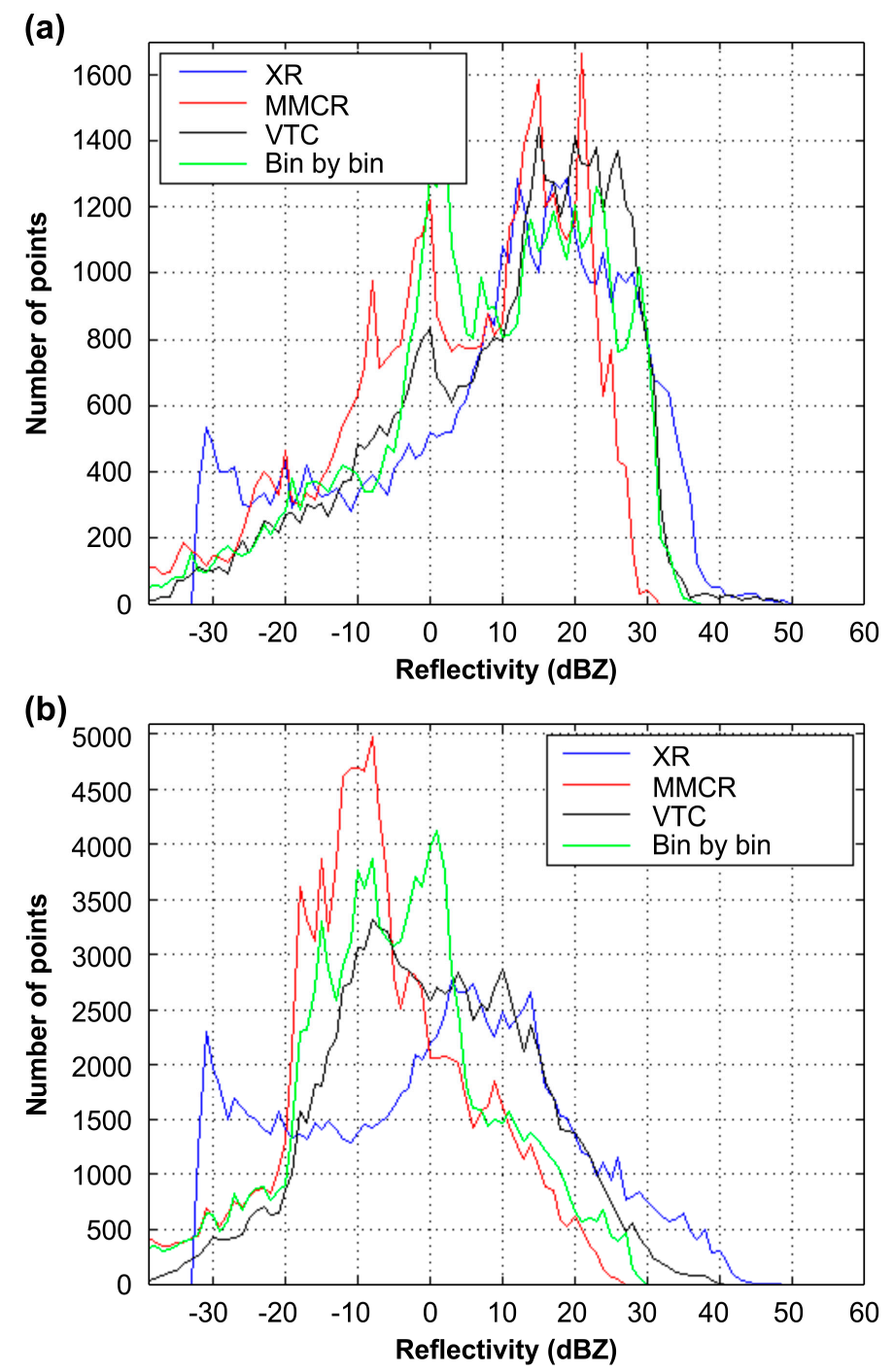

Figure 5. Histogram of reflectivities in 3-km constant altitude plan position indicator (CAPPI) of millimeter-wavelength cloud radar (MMCR) and X-band weather radar (XR) before attenuation correction, after VTC attenuation correction, and after bin-by-bin attenuation correction at (a) 13:22 on 26 July 2010 and (b) 9:18 on 28 July 2010.

The number of relatively larger reflectivity points in the MMCR data before correction were significantly lower than those in the XR data, but increased notably after correction, with the VTC correction resulting in a greater increase than the bin-by-bin method (Figure 5). After attenuation correction with the VTC method, the mean reflectivity factor of the MMCR increased significantly (by $8.98 \mathrm{~dB}$ ) and becomes closer to that of the XR (Table 3); furthermore, the correlation between the 3- $\mathrm{km}$ CAPPI data of the MMCR and the XR increased notably (by 0.2). Again, the improvement using the VTC method was greater than that using the bin-by-bin method. It is important to note that because this study focuses on the correction of the reflectivities already obtained by the MMCR, and the VTC method presented here was prohibited from performing corrections where no echo was detected by the MMCR. However, without this limitation, the VTC method can recreate echoes detected by the XR but not by the MMCR. In other words, the data fusion of the two radars can be achieved. 


\begin{tabular}{lcccc} 
RR & Statistics & Reflectivities of echoes detected by both radars \\
\cline { 2 - 5 } & & Mean/dBZ & Std Dev/dBZ & Correlation \\
\hline \multirow{2}{*}{ MMCR } & Before correction & -6.53 & 18.51 & 0.41 \\
\cline { 2 - 5 } & After VTC & 2.45 & 19.47 & 0.61 \\
\cline { 2 - 5 } & After bin-by-bin & -4.16 & 20.62 & 0.48
\end{tabular}

a.Millimeter-wavelength cloud radar (MMCR) and X-band weather radar of the World Meteorological Organization (WMO) $8^{\text {th }}$ radiosondes inter-comparison and integrated remote instruments experiment (July 2010, Guangzhou Province, China)

\subsection{Impact of distance between radars}

The VTC method can be used for the attenuation correction of shorter wavelength radar data assisted by longer wavelength radar data. The locations of the shorter and longer wavelength radar do not need to be the same; however, factors including the layout and wavelength differences of the two radars can affect the accuracy of the VTC method.

It is assumed that variation trends in reflectivity along the same path but detected by the radars of different wavelengths would be similar without the impact of attenuation. However, as discussed, the resolution volume difference caused by different beam widths and pulse repetition frequencies (PRF) results in "common volume" reflectivity differences. These reflectivity differences increase with increasing distance between radars, especially when the weather system is convective and has high spatial variations in reflectivity. At the same time, the distance between radars can change the "gaps" between adjacent elevations in volume scan data. This can also introduce errors into CAPPI data through interpolation, which result in errors within the final VTC method. Therefore, when using the VTC correction, the distance between the two radars should be as short as possible. However, if the "blind" detection zone above the longer wavelength radar is within the detection area of the shorter wavelength radar, then the accuracy of the VTC method will also be influenced because there will be no data to extract the variation trend in the "blind" detection zone. Therefore, the VTC method works best when the longer wavelength radar is very close to the shorter-wavelength radar, or located just near the edge of the observation range of the shorter wavelength radar.

\subsection{Impact of radar wavelength differences}

As the difference in wavelength between the two radars increases, the 
differences in "common" volume reflectivities due to non-Rayleigh scattering also increase. However, if the difference between the wavelengths of the two radars is too low, the variation trend extracted from the reflectivities of the longer wavelength radar will be useless because the attenuations suffered by the two radars will be almost the same. Therefore, the VTC method works best when the wavelengths of the two radars are adjacent in the standard wavelength list (e.g., $\mathrm{Ka}$ and $\mathrm{X}$ band, $\mathrm{X}$ and $\mathrm{C}$ band, $\mathrm{C}$ and $\mathrm{S}$ band).

\subsection{Impacts of observation time and clutter}

Observation time mismatches can result in "common" volume reflectivity differences, especially when the temporal variability of the weather system is high. To address this problem, radars should operate at high scan rates. The different clutters suffered by radars can also result in reflectivity differences and effective clutter mitigation must also be performed before the VTC attenuation correction.

\section{Conclusions}

High-quality MMCR data are the basis of quantitative applications. To reduce attenuation-based error in MMCR data, and to address problems of unstable correction, which are inherent in conventional attenuation correction methods, a new correction methodology using variation trends as constraint was developed and evaluated. On the basis of the results, a number of conclusions can be drawn.

Compared with centimeter-wavelength radar, MMCR can detect weaker echoes; however, if intense echoes are in the wave propagation path, the reflectivity factors of MMCR will be lower than those of centimeter-wavelength radar, mainly because of attenuation. The proposed VTC method can correct the reflectivity factors of MMCR by using variation trends in the reflectivities of $\mathrm{XR}$ as a constraint. In this study, after correction with the VTC method, the mean value of MMCR reflectivities and the correlation with the XR reflectivities both increased, where the correction amplitude was larger than that achieved using the bin-by-bin correction method. Furthermore, the variation details in the MMCR reflectivity data before correction were effectively retained. The accuracy of the VTC method is affected mainly by the distance and wavelength differences between radars.

Future development of the VTC method will focus on the collection of additional radar data and the analysis errors caused by layout and the wavelength differences between the two radars. Data fusion of the reflectivities in MMCR and XR data using the VTC method will also be investigated in order to improve the retrieval accuracy of macro and micro cloud parameters.

\section{ACKNOWLEDGMENTS}

This research was supported by the National Natural Science Foundation of China (Grant no. 41174164 and 61473310) and the Open Fund of Jiangsu Key Laboratory 
of Meteorological Observation and Information Processing (Grant no. KDXS1306).

\section{REFERENCES}

[1] Moran, K. P., B. E. Mariner, M. J. Post, R. A. Kropfli, D. C. Welsh, and K. B. Widener, "An unattended cloud-profiling radar for use in climate research," Bull. Amer. Meteor. Soc., vol. 79, no. 3, pp. 443-455, 1998.

[2] Kropfli, R. A., and R. D. Kelly, "Meteorological research applications of MM-wave radar," Meteor. Atmos. Phys., vol. 59, no. 2, pp. 105-121, 1996.

[3] Lhermitte, R., "Attenuation and Scattering of Millimeter Wavelength Radiation by Clouds and Precipitation," J. Atmos. Oceanic Technol., vol. 7, no. 6, pp. 464-479, 1990.

[4] Kollias, P., E. E. Clothiaux, M. A. Miller, B. A. Albrecht, G. L. Stephens, and T. P. Ackerman, "Millimeter-wavelength radars," Bull. Amer. Meteor. Soc., vol. 88, no. 10, pp. 1608-1624, 2007.

[5] Borque, P., P. Kollias, S. Giangrande, "First Observations of Tracking Clouds Using Scanning ARM Cloud Radars," J. Appl. Meteor. Climatology, vol. 53, no. 12, pp. 2732-2746, 2014.

[6] Kollias, P., and B. Albrecht, "The Turbulence Structure in a Continental Stratocumulus Cloud from Millimeter-Wavelength Radar Observations," J. Atmos. Sci., vol. 57, no. 15, pp. 2417-2434, 2000.

[7] Kalesse, H., and P. Kollias, " Climatology of High Cloud Dynamics Using Profiling ARM Doppler Radar Observations ," J. Climate, vol. 26, no. 17, pp. 6340-6359, 2013.

[8] Lawson, R. P., and P. Zuidema, "Aircraft Microphysical and Surface-Based Radar Observations of Summertime Arctic Clouds," J. Atmos. Sci., vol. 66, no. 12, pp. 3505-3529, 2009.

[9] Lu, Y. H., K. Aydin, E. E. Clothiaux, and J. Verlinde, "Retrieving Cloud Ice Water Content Using Millimeter- and Centimeter-Wavelength Radar Polarimetric Observables," J. Appl. Meteor. Climatology, vol. 54, no. 3, pp. 596-604, 2015.

[10] Matrosov, S. Y., M. D. Shupe, and I. V. Djalalova, "Snowfall Retrievals Using Millimeter-Wavelength Cloud Radars," J. Appl. Meteor. Climatology, vol. 47, no. 3, pp. 769777, 2008.

[11] Ulbrich, C. W., "The Accuracy of Rainfall Rate Measurement by Tunable Millimeter-Wavelength Radar," J. Climate Appl. Meteor., vol. 22, no. 10, pp. 1824-1828, 1983.

[12] Hildebrand, P. H., "Iterative correction for attenuation of $5 \mathrm{~cm}$ radar in rain," J. Appl. Meteor., vol. 17, no. 4, 508-514, 1978.

[13] Hitschfeld, W., and J. Bordan, "Errors inherent in the radar measurement of rainfall at attenuating wavelengths," J. Meteor., vol. 11, no. 1, pp. 58-67, 1954.

[14] Delrieu, G., S. Caoudal, and J. D. Creutin, "Feasibility of Using Mountain Return for the Correction of Ground-Based X-Band Weather Radar Data," J. Atmos. Oceanic Technol., vol. 14, no. 3, pp. 368-385, 1997.

[15] Meneghini, R., J. Eckerman, and D. Atlas, "Determination of rain rate from a spaceborne radar using measurements of total attenuation," IEEE Trans. Geosci. Remote Sens., vol. 21, no.1, pp. 34-43, 1983.

[16] Tuttle, J. D., and R. E. Rinehart, "Attenuation Correction in Dual-Wavelength Analyses.," J. Climate Appl. Meteor., vol. 22, no. 11, pp. 1914-1921, 1983.

[17] Seto, S., and T. Iguchi, "Intercomparison of Attenuation Correction Methods for the GPM Dual-Frequency Precipitation Radar," J. Atmos. Oceanic Technol., vol. 32, no. 5, pp. 915-926, 2015.

[18] Carey, L. D., S. A. Rutledge, D. A. Ahijevych, and T. D. Keenan, "Correcting propagation effects in C-band polarimetric radar observations of tropical convection using differential propagation phase," J. Appl. Meteor., vol. 39, no. 9, pp. 1405-1433,2000. 
[19] Ryzhkov, A., and D. S. Zrnic, "Precipitation and attenuation measurements at a 10-cm wavelength," J. Appl. Meteor., vol. 34, no. 10, pp. 2121-2134, 1995.

[20] Smyth, T. J., and A. J. Illingworth, "Correction for attenuation of radar reflectivity using polarization data," Quart. J. Roy. Meteor. Soc., vol. 124, no. 551, pp. 2393-2415, 1998.

[21] Jameson, A. R., "The effect of temperature on attenuation-correction schemes in rain using polarization propagation differential phase shift," J. Appl. Meteor., vol. 31, no. 9, pp. 11061118, 1992.

[22] Bringi, V. N., T. D. Keenan, and V. Chandrasekar, "Correcting C-band radar reflectivity and differential reflectivity data for rain attenuation: A self-consistent method with constraints," IEEE Trans. Geosci. Remote Sens., vol. 39, no. 9, pp. 1906-1915, 2001.

[23] Park, S.-G., V. N. Bringi, V. Chandrasekar, M. Maki, and K. Iwanami, "Correction of Radar Reflectivity and Differential Reflectivity for Rain Attenuation at X Band. Part I: Theoretical and Empirical Basis," J. Atmos. Oceanic Technol., vol. 22, no. 11, pp. 1621-1632, 2005.

[24] Ryzhkov, A., and D. S. Zrnic, "Assessment of rainfall measurement that uses specific differential phase," J. Appl. Meteor., vol. 35, no. 1996, pp. 2080-2090, 1996.

[25] You, C. H., Lee, D. I., and Kang, M. Y., "Rainfall estimation using specific differential phase for the first operational polarimetric radar in korea," Advances in Meteorology, vol. 2014, no. 1, pp. 1-10, 2014.

[26] Testud, J., and P. Amayenc, "Stereoradar Meteorology: A Promising Technique for observation of Precipitation from a Mobile Platform," J. Atmos. Oceanic Technol., vol. 6, no. 1, pp. 89-108, 1989.

[27] Weinman, J. A., R. Meneghini, and K. Nakamura, "Retrieval of precipitation profiles from airborne radar and passive radiometer measurements, comparison with dual-frequency radar measurements," J. Appl. Meteor., vol. 29, no. 10, pp. 981-993, 1990.

[28] Cummingsa, R. J., A. R. Holt, and M. Kitchen, "Using microwave links to adjust the radar rainfall field," Adv. Water Resour., vol. 32, no. 7, pp. 1003-1010, 2009.

[29] Krämer, S., H. R. Verworn, and A. Redder. "Improvement of X-band radar rainfall estimates using a microwave link," Atmospheric Research, vol. 77, no. 1, pp. 278-299, 2005.

[30] Trömel, S., M. Ziegert, A. V. Ryzhkov, C. Chwala, and C. Simmer, "Using Microwave Backhaul Links to Optimize the Performance of Algorithms for Rainfall Estimation and Attenuation Correction," J. Atmos. Oceanic Technol., vol. 31, no. 8, pp. 1748-1760, 2014.

[31] Ostrometzky, Jonatan, D. Cherkassky, and H. Messer. "Accumulated Mixed Precipitation Estimation Using Measurements from Multiple Microwave Links," Advances in Meteorology, 2015, 1-9, 2015.

[32] Chandrasekar, V., Willie, D., Wang, Y., and Lim, S., "Attenuation margin requirements in a networked radar system for observation of precipitation," Geoscience and Remote Sensing Symposium, 2009 IEEE International, IGARSS 2009 (Vol.2, pp.II-957-II-959). IEEE.

[33] Stepanenko, V. D., G. G. Schukin, L. P. Bobylev, and S. Y. Matrosov, "Microwave Radiometry in Meteorology," Gidrometeoizdat, pp. 284, 1987.

[34] Nakamura, K., and H. Inomata, "Non-Rayleigh Scattering Effect in Rain Observations by X- and Ka-band Dual-Wavelength Radar," J. Atmos. Oceanic Technol., vol. 8, no. 3, pp. 352$362,1991$.

[35] Chandrasekar, V., S. Lim, and E. Gorgucci, "Simulation of X-Band Rainfall Observations from S-Band Radar Data," J. Atmos. Oceanic Technol., vol. 23, no. 9, pp. 1195-1205, 2006.

[36]Borowska L., A. Ryzhkov, D. Zrnic, C. Simmer, and R. Palmer, "Attenuation and Differential Attenuation of 5-cm-Wavelength Radiation in Melting Hail," J. Appl. Meteor. Climatology, vol. 50, no. 1, pp. 59-76, 2011.

[37] Vulpiani, G., Marzano, F. S., Chandrasekar, V., and Lim, S., "Constrained iterative technique with embedded neural network for dual-polarization radar correction of rain path attenuation," Geoscience \& Remote Sensing IEEE Transactions on, vol. 43, no. 10, pp. 
2305-2314, 2005.

[38] Gorgucci, E., and Baldini, L., "Attenuation and differential attenuation correction of c-band radar observations using a fully self-consistent methodology," IEEE Geoscience \& Remote Sensing Letters, vol. 4, no. 2, pp. 326-330, 2007.

[39] Huang, X. Y., Y. W. Fan, F. Li, H. Xiao, and X. Zhang, "The attenuation correction for a 35GHz ground-based cloud radar," J. Infrared Millim. Waves, vol. 32, no. 4, pp. 325-330, 2013 (in Chinese).

(C) 2016 by the authors; licensee Preprints, Basel, Switzerland. This article is an open access article distributed under the terms and conditions of the Creative Commons by Attribution (CC-BY) license (http://creativecommons.org/licenses/by/4.0/). 